\title{
Measurement of the long-range beam-beam effect at injection, and design for a compensator in RHIC
}

\author{
W. Fischer, R. Alforque, H.C. Hseuh, R. Lambiase, C.J. Liaw, \\ G. Miglionico, T. Russo, BNL \\ J.-P. Koutchouk, F. Zimmermann, CERN \\ T. Sen, FNAL
}

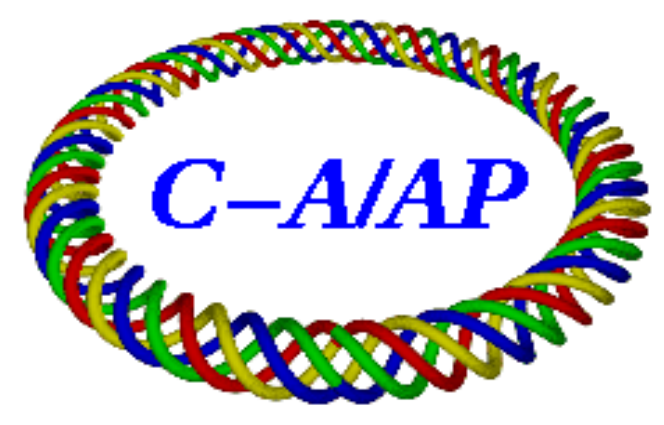

Collider-Accelerator Department Brookhaven National Laboratory Upton, NY 11973 
BNL C-A/AP/236

\title{
Measurement of the long-range beam-beam effect at injection, and design for a compensator in RHIC
}

\author{
W. Fischer, R. Alforque, H.C. Hseuh, B. Lambiase, \\ C.J. Liaw, G. Miglionico, T. Russo, BNL \\ J.-P. Koutchouk, F. Zimmermann, CERN \\ T. Sen, FNAL
}

April 17, 2006

\begin{abstract}
At large distances the electromagnetic field of a wire is the same as the field produced by a bunch. Such a long-range beam-beam wire compensator was proposed for the LHC, and single beam tests with wire compensators were successfully done in the SPS. RHIC offers the possibility to test the compensation scheme with colliding beams. We report on measurements of beam loss as a function of transverse separation at injection, and present a design for a long-range wire compensator in RHIC.
\end{abstract}

\section{Introduction}

In colliders that store particles and antiparticles in the same beam pipe all bunches encounter the other beam not only at the designated interaction points but also at other locations*. At these other locations the beams are separated as much as possible, but the bunches are still affected by the electromagnetic field of the other beam. Even in colliders with two separate rings, long-range interactions can be encountered if the bunches are spaced by only a small distance. It is then difficult to separate the beams coming out of an interaction region fast enough. Two concepts were proposed to compensate longrange beam-beam interactions: electron lenses for the Tevatron $[1,2]$ and wires for the LHC $[3,4]$. Electron lenses were studied in the Tevatron, and wires were tested in the SPS with a single beam [5]. Here we consider tests in RHIC for wire compensators.

Like the LHC, RHIC has two separate beam pipes, and possible long-range interactions are localized in the interaction regions. In contrast, in the Tevatron long-range interactions are distributed around the circumference, which makes compensation more complex $[6,7]$. In RHIC, with a bunch spacing of 3 accelerating rf buckets (108 ns, the current minimum) or more, there are no parasitic beam-beam collisions in the interaction region (see Fig. 1). With eRHIC, in particular the ring-ring option [8], it is planned to increase the number of bunches, leading to a bunch spacing as short as only $1 \mathrm{rf}$ bucket (36 ns). In this case the bunches would encounter 2 parasitic beam-beam interactions on each side of the

${ }^{*}$ This is strictly true only if there are more bunches than half the designated interaction points, a condition usually fulfilled. 


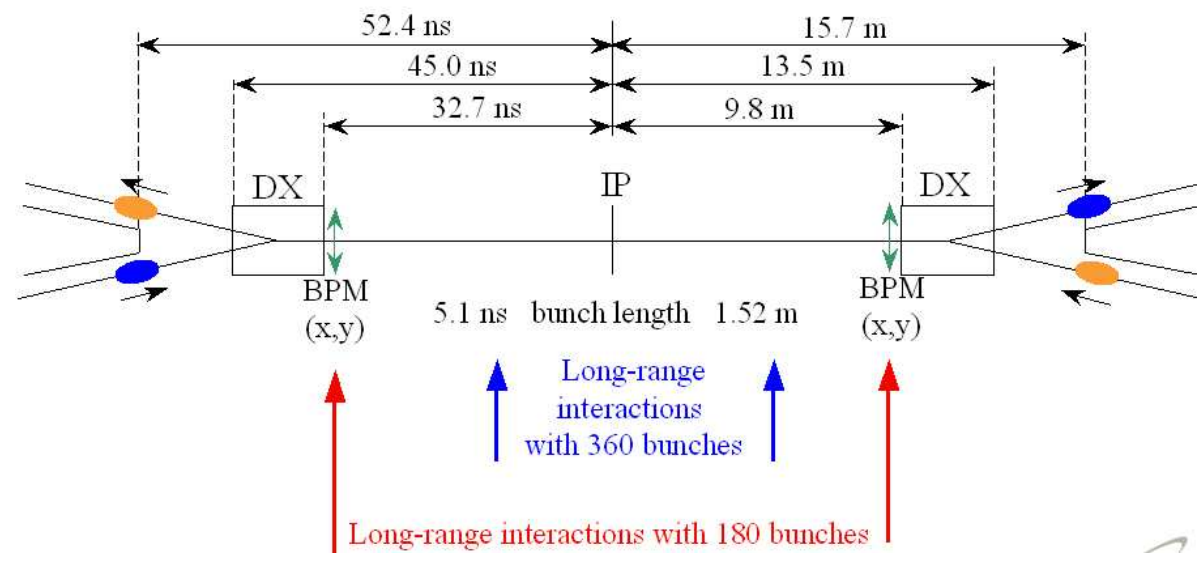

Figure 1: RHIC interaction region. Bunches are separated after reaching the DX magnets. The stated full bunch length is for the $200 \mathrm{MHz}$ storage rf system.

interaction point [9] and a long-range beam-beam compensation may be beneficial to the beam and luminosity lifetimes.

To test a long-range beam-beam compensator in RHIC, there must be an observable effect from long-range interactions. We report on beam loss measurements at injection, in which the vertical separation of 2 bunches was varied. Simulations were carried out in an attempt to reproduce the observations. We present a design for a long-range compensator, to be installed in RHIC in 2006.

\section{Observation of long-range beam-beam effects at injection}

To test a long-range beam-beam compensator in RHIC, a single long-range interaction must produce a measurable effect (see Sec. 3.1). As a first test, the beam loss rates of two colliding proton bunches was observed as a function of vertical separation at injection. The main parameters of this test are shown in Tab. 1, and the transverse tunes in Fig. 2. A total of 4 scans were done, while a scan consistent of the injection of two new bunches and the variation of the vertical separation.

Scan 1. In the first scan, the long-range interaction took place at the nominal interaction point with $s=0 \mathrm{~m}$ (see Fig. 1). The Blue beam was moved to change the vertical separation. In this scan both the Blue and the Yellow beam losses increased with decreasing separation (Fig. 3), although the Blue losses were much larger than the Yellow losses at separations below $4 \sigma$. The Blue and Yellow bunches had different transverse tunes (see Tab. 1 and Fig. 2), and the Blue tunes were closer to a $4^{\text {th }}$ order resonance.

Scan 2. In the second scan the long-range interaction took place at $s=10.6 \mathrm{~m}$, at the entrance to the DX beam separation dipole (Fig. 4). This is the location where long-range interaction would take place if the bunch spacing in RHIC were 2 accelerating rf buckets (72 ns). It is also the location that can be used to test a long-range compensator, placed just after Q3, in store (see Sec. 3.1). In this case too, the Blue beam was moved. The 
Table 1: Main parameters for the RHIC test at injection.

\begin{tabular}{|c|c|c|}
\hline quantity & unit & value \\
\hline proton energy & $\mathrm{GeV}$ & 24.3 \\
\hline relativistic $\gamma$ & $\ldots$ & 26.3 \\
\hline bunches per beam & $\ldots$ & 1 \\
\hline bunch intensity & $10^{11}$ & $1-2$ \\
\hline norm. emittances $\epsilon_{x}, \epsilon_{y}(95 \%)$ & $\mathrm{mm} \operatorname{mrad}$ & 20,13 \\
\hline location of long-range interaction & $\mathrm{m}$ from IP & $0.0,10.6$ \\
\hline transverse tunes $\left(Q_{x}, Q_{y}\right)$, scan 1 & $\ldots$ & $\mathrm{B}(0.733,0.727) \mathrm{Y}(0.727,0.723)$ \\
\hline scan 2 & $\ldots$ & $\mathrm{B}(0.735,0.722) \mathrm{Y}(0.728,0.723)$ \\
\hline scan 3 & $\ldots$ & $\mathrm{B}(0.738,0.725) \mathrm{Y}(0.727,0.722)$ \\
\hline scan 4 & $\ldots$ & $\mathrm{B}(0.739,0.727) \mathrm{Y}(0.726,0.739)$ \\
\hline vertical separation & $\mathrm{mm}$ & $10-0$ \\
\hline & $\sigma$ & $11-0$ \\
\hline
\end{tabular}

tunes of both beams are close to those in scan 1. The Blue beam shows losses increasing with separations of 6 or less $\sigma$, the Yellow beam shows smaller losses than in scan 1 .

Scan 3. In scan 1 and scan 2 the Blue beam is moved vertically, and in both cases, the Blue beam losses were larger than the Yellow beam losses. To exclude the possibility that the Blue losses stem from tune changes as a result of the orbit movements, a third scan was done, in which the Yellow beam is moved, and the other conditions are close to those in scan 2. This is shown in Fig. 5. Here again the Blue losses are larger than the Yellow losses, indicating that the changes in the Blue loss rates in scan 1 and scan 2 are not due to the Blue orbit changes, but due to the increasing effect of the beam-beam interaction.

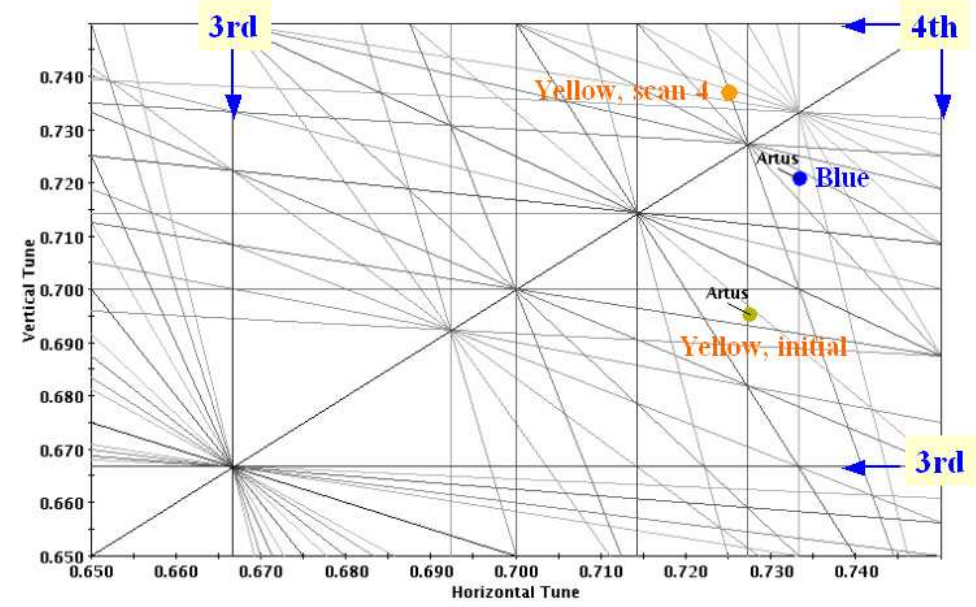

Figure 2: Tune diagram with Blue and Yellow tunes. The Yellow tunes were move for scan 4. 


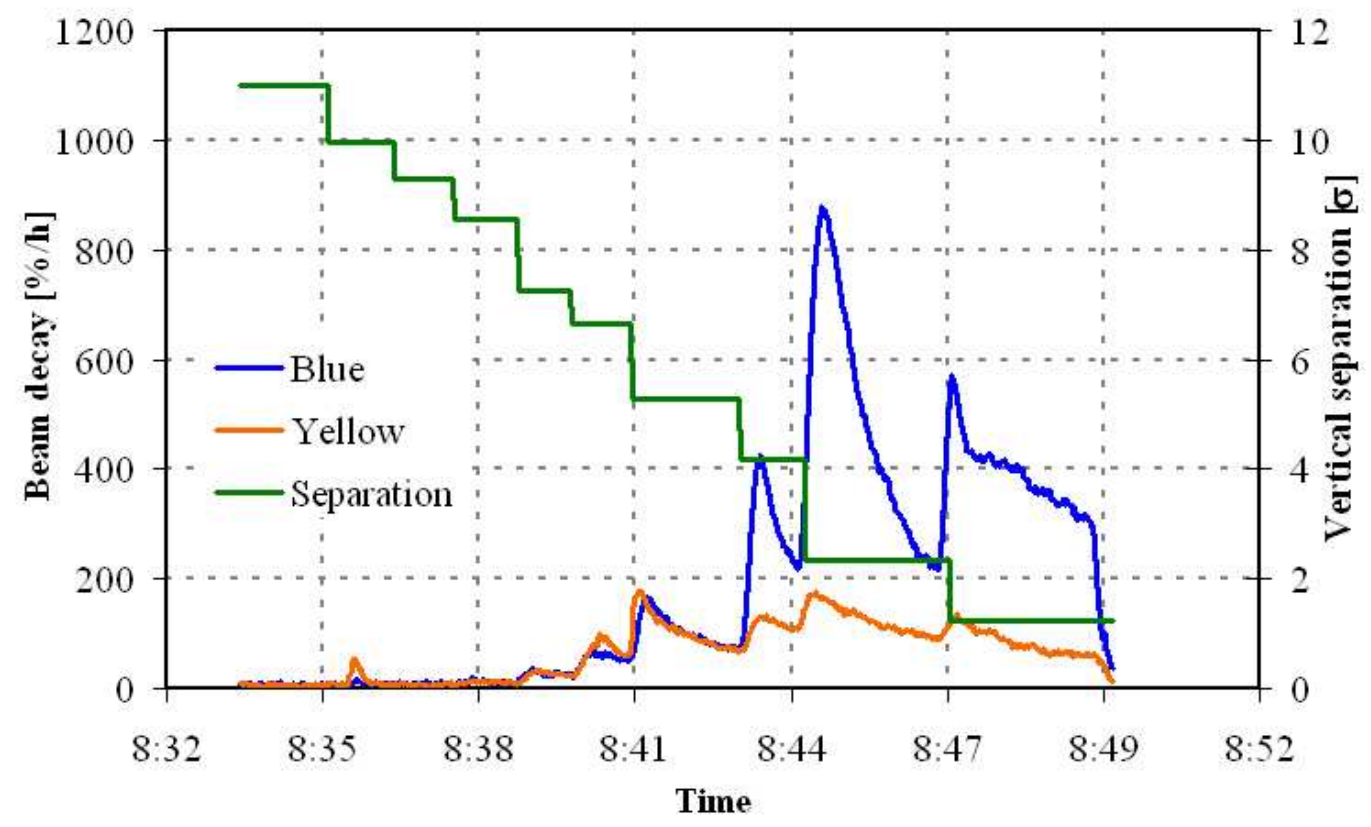

Figure 3: Scan 1. Long-range beam-beam interaction at $s=0 \mathrm{~m}$, Blue beam is moved.

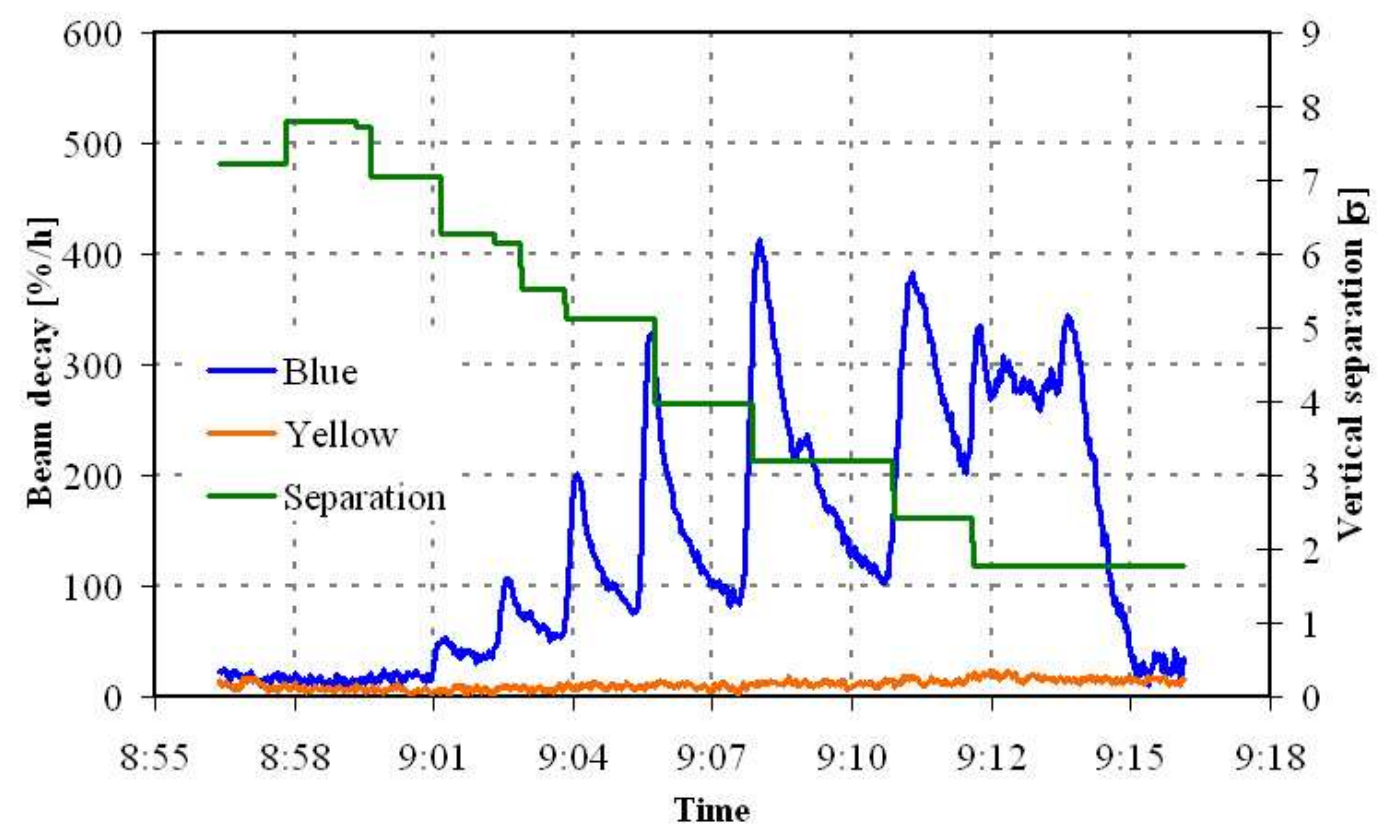

Figure 4: Scan 2. Long-range beam-beam interaction at $s=10.6 \mathrm{~m}$, Blue beam is moved. 


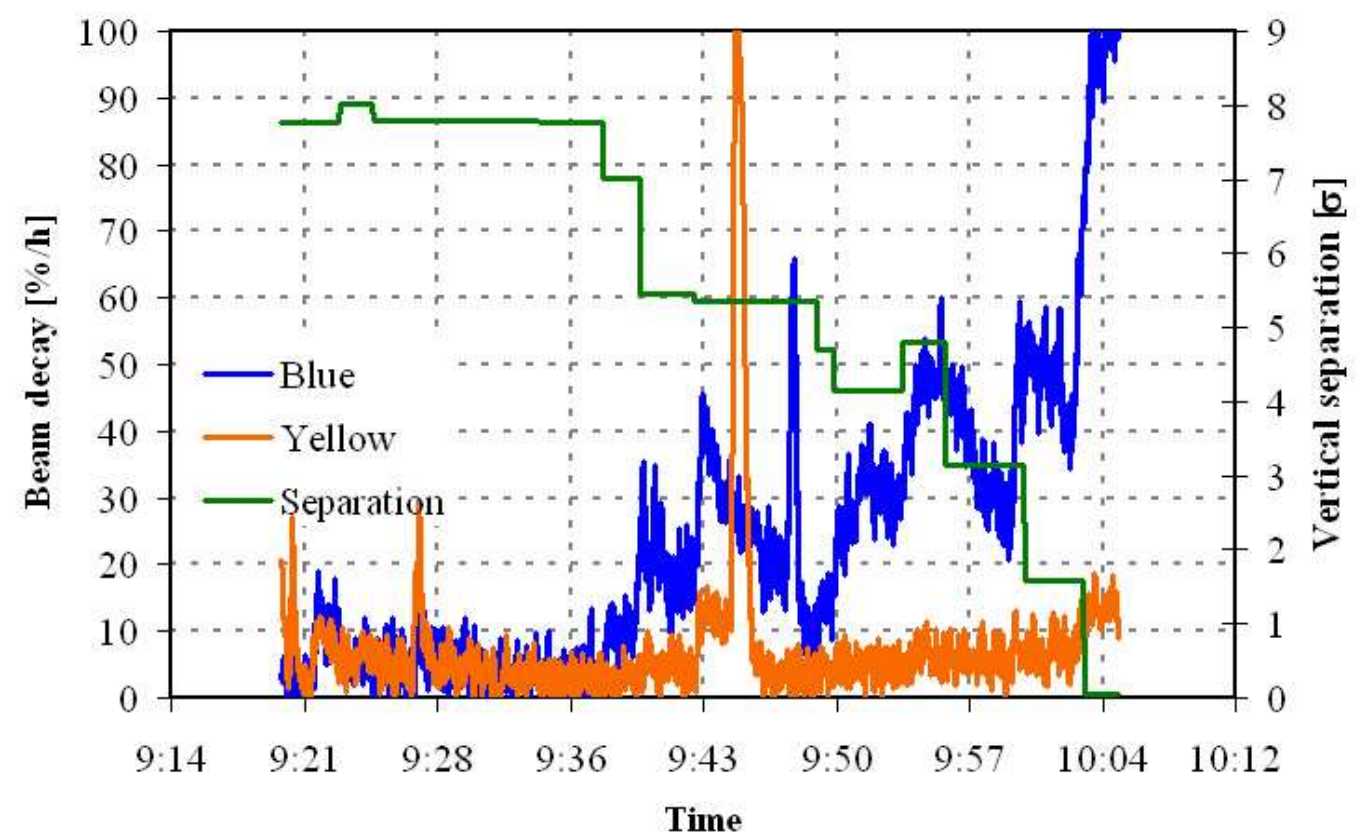

Figure 5: Scan 3. Long-range beam-beam interaction at $s=10.6 \mathrm{~m}$, Yellow beam is moved.

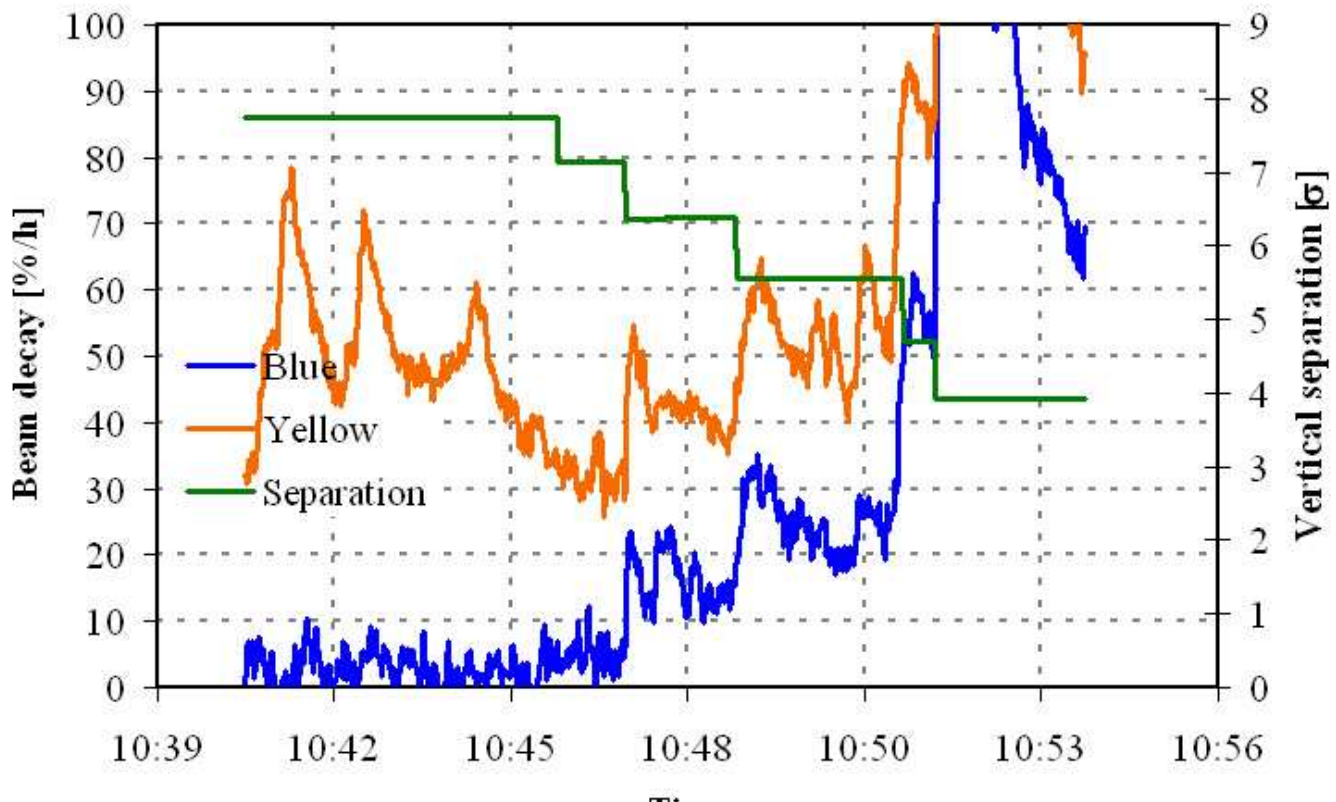

Time

Figure 6: Scan 4. Long-range beam-beam interaction at $s=10.6 \mathrm{~m}$, Blue beam is moved. New working point for Yellow beam. 
Scan 4. In scans 1 through 3, the Blue beam losses were larger than the Yellow beam losses. But the Blue tunes were different from the Yellow tunes (see Fig. 2). For scan 4 the Yellow tunes were moved to the Blue tunes, mirrored at $Q_{x}=Q_{y}$, and again the Blue beam was moved. The initial losses of the Yellow beam are significant (see Fig. 6), and attempts to improve the beam lifetime through small changes in the tunes did not succeed. In the following scan of the vertical separation. the losses of both beams are enhanced when the beam separation is smaller than $6 \sigma$.

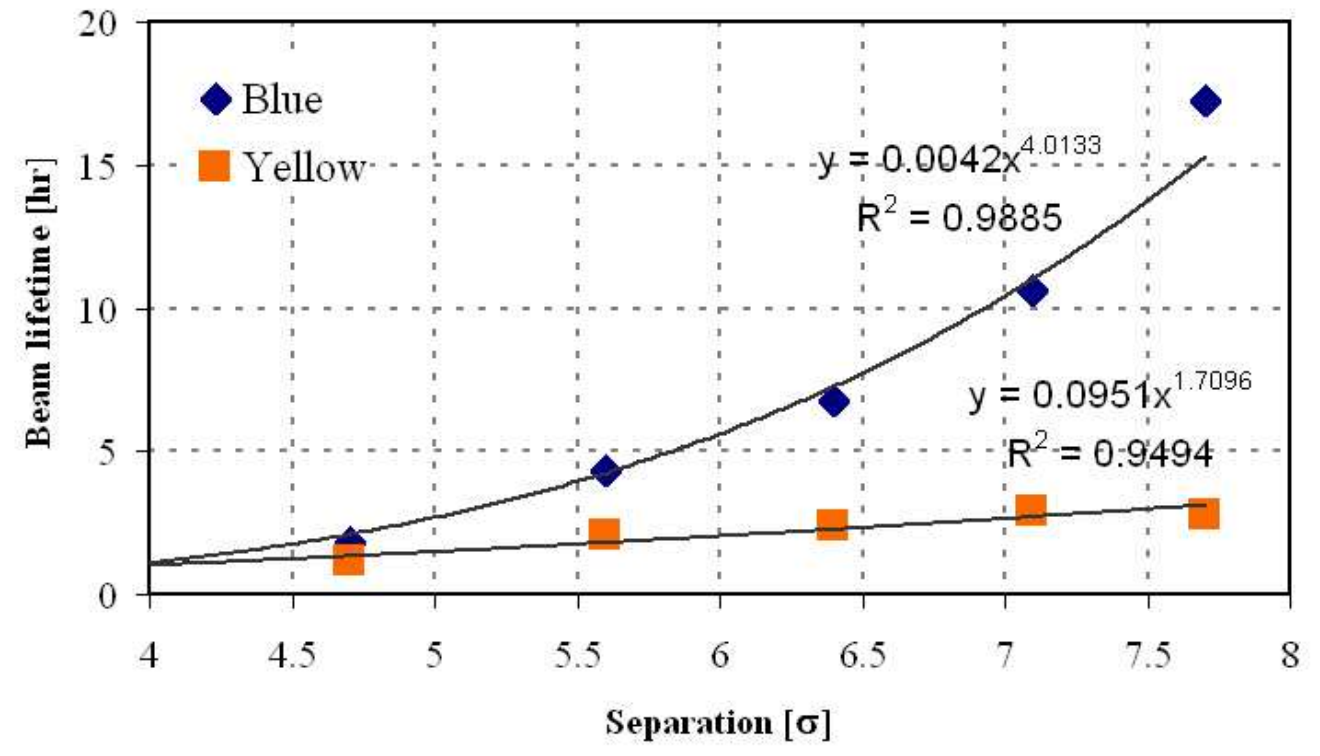

Figure 7: Blue and Yellow beam lifetime as a function of vertical separation in scan 4, and fits to an exponential function.

From these scans one can conclude, firstly, that a long-range beam-beam effect is observable in RHIC at injection, and secondly, that the effect is very sensitive to the transverse tunes. Fig. 7 shows the fitted beam lifetime as a function of the vertical beam separation, for the beam loss rates observed in scan 4. For the Blue beam the lifetime $\tau$ is proportional to $d^{4}$, where $d$ is the distance between the two beams. For the Yellow beam we have $\tau \propto d^{2}$. In the SPS $\tau \propto d^{5}$ was observed [10], in the Tevatron $r \propto d^{3}[11]$.

\section{Compensator design}

The RHIC long-range compensator design is based on experience gained with the constructed and tested SPS units. Design considerations are:

- location in ring

- integrated strength

- wire temperature in operation

- positioning range and accuracy 
- power supply requirements

- controls and diagnostics

We discuss these requirements below.

\subsection{Location in the ring}

For a successful compensation the phase advance between the long-range interaction and the compensator should be as small as possible, while a few degrees are tolerable. In Ref. [12] a phase error of up to 10 degrees was found to be acceptable in simulations. In a store lattice with small $\beta^{*}$ the $\beta$-function in the triplets is large and the phase advance across the triplet small. Thus it is possible to place a wire after the triplet to compensate for a long-range beam-beam interaction in the interaction region.

Fig. 8 shows one side of a RHIC interaction region. A single long-range beam-beam interaction can be placed anywhere between the DX magnets. With a bunch spacing of 3 accelerating buckets, 2 long-range interactions can be placed between DX magnets (see also Fig. 1). While the long-range interaction must take place in a pipe common to both beams, the compensator can only be located in a single-beam pipe. The space between the magnets Q3 and Q4 has a warm beam pipe, and can accommodate a compensator if it does not interfere with other equipment.

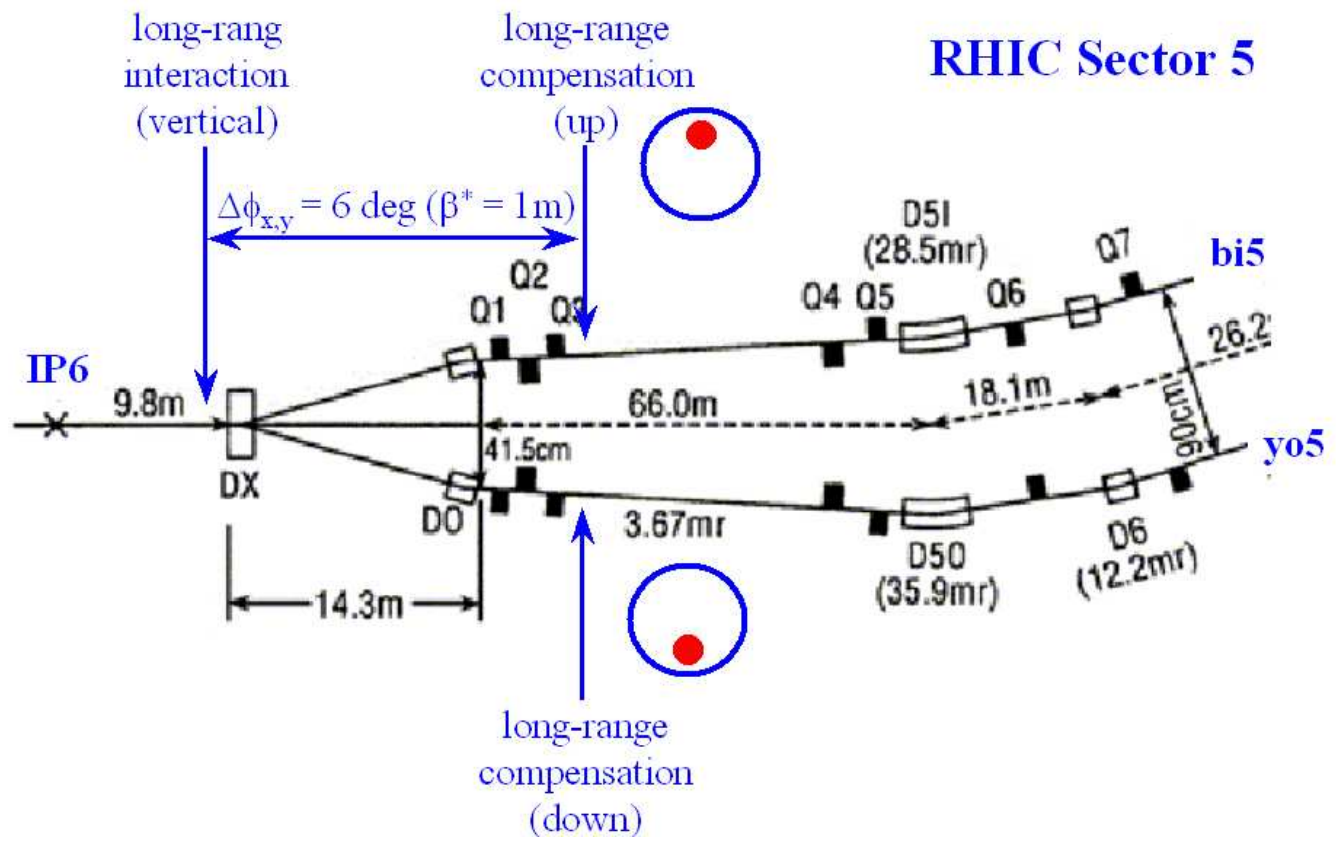

Figure 8: Location of the long-range beam-beam interaction and compensators for a beam test in RHIC.

Tab. 2 shows the phase advances between interaction point IP6 and a number of elements in sector 5 . Only for lattices with $\beta^{*} \leq 1.0 \mathrm{~m}$ the phase advance between the entrance to the DX and the exit of Q3 is small enough to expect an effective long-range 
compensation. No other combination of long-range interaction and compensator location was found with a phase advance of only a few degrees.

Table 2: Phase advances in the interaction region for different lattices. Shown are the phase advance between IP6 and selected locations in sector 5 (ring locations bi5 and yo5). The phase advances are the same for both the Blue and Yellow ring.

\begin{tabular}{lc|cccc|c}
\hline \hline Lattice & $\begin{array}{c}\beta^{*} \\
{[\mathrm{~m}]}\end{array}$ & $\begin{array}{c}s=0 \mathrm{~m} \\
\text { IP6 } \\
{[\mathrm{deg}]}\end{array}$ & $\begin{array}{c}s=9.8 \mathrm{~m}(\mathrm{in}) \\
{[\mathrm{deg}]}\end{array}$ & $\begin{array}{c}s=38.5 \mathrm{~m} \\
\mathrm{Q} 3(\mathrm{out}) \\
{[\mathrm{deg}]}\end{array}$ & $\begin{array}{c}s=73.0 \mathrm{~m} \\
\text { Q4(in) } \\
{[\mathrm{deg}]}\end{array}$ & $\begin{array}{c}\Delta \phi(\mathrm{DX}-\mathrm{Q} 3) \\
{[\mathrm{deg}]}\end{array}$ \\
\hline \multirow{3}{*}{ injection } & 10.0 & 0.0 & 39.0 & 76.8 & 119.4 & 37.8 \\
store & 5.0 & 0.0 & 59.4 & 82.8 & 106.6 & 23.4 \\
store & 3.0 & 0.0 & 69.5 & 85.7 & 102.6 & 16.2 \\
store & 1.0 & 0.0 & 83.1 & 88.7 & 96.5 & 5.7 \\
store & 0.85 & 0.0 & 84.2 & 88.9 & 95.8 & 4.7 \\
store & 0.5 & 0.0 & 87.8 & 90.4 & 88.2 & 2.5 \\
\hline & & \multicolumn{5}{|c}{ vertical } \\
injection & 10.0 & 0.0 & 39.3 & 78.3 & 195.8 & 38.9 \\
store & 5.0 & 0.0 & 59.8 & 84.2 & 207.7 & 24.5 \\
store & 3.0 & 0.0 & 69.8 & 86.8 & 220.3 & 16.9 \\
store & 1.0 & 0.0 & 83.0 & 88.8 & 246.7 & 5.7 \\
store & 0.85 & 0.0 & 85.0 & 89.6 & 249.5 & 4.7 \\
store & 0.5 & 0.0 & 86.0 & 88.9 & 95.4 & 2.9 \\
\hline \hline
\end{tabular}

Only the interaction points IP6 (STAR experiment) and IP8 (PHENIX experiment) can accommodate a $\beta^{*}$ of $1 \mathrm{~m}$ or less. To compensate the long-range beam-beam effect in both beams, with a beam-beam interaction near the DX entrance, the two compensators must be placed side-by-side at the Q3 exit. In IR8 the long straight section of the outgoing beam between Q3 and Q4 houses the collimators, and installation of additional equipment is not possible.

We have therefore chosen sector 5 (ring locations bi5 and yo5) for the installation of the compensators (Fig. 8). The longitudinal space from $s=39.064 \mathrm{~m}$ to $s=42.723 \mathrm{~m}$ from the IP (3.659 $\mathrm{m}$ of space) has been reserved in both rings. This allows for a compensator with a wire length of $L=2.5 \mathrm{~m}$, as well as bellows and flanges. Fig. 9 shows the 2 compensators side-by-side. This assembly is next to the cryostat that houses the Q3 magnets.

The beams cross in every IR horizontally, and it is easier to control the vertical separation. For $\beta^{*}=1$, a beam energy of $100 \mathrm{GeV}$, and and emittance of $20 \mathrm{~mm} \cdot \mathrm{mrad}$, the maximum rms beam size in the triplets is $\sigma=6.5 \mathrm{~mm}$. The limiting aperture comes from the beam pipe connecting to Q3, with a radius of $60 \mathrm{~mm}$. This gives an aperture of $9 \sigma$. A vertical displacement of 1-2 $\sigma$, or $6-13 \mathrm{~mm}$ may be possible, allowing for a separation of 6-12 $\sigma$ at the long-range interaction point. Prior collimation may be needed before the beams can be separated by the largest values. In operation, beams are displaced by only $\pm 3 \mathrm{~mm}$. One long-range compensator will be placed above, and one below the beam axis. In operation, the Blue beam is kept above IP6 and the Yellow beam below IP6 when the 
beams are separated. We will therefore place the Blue long-range compensator below the nominal orbit, and the Yellow compensator above the nominal orbit.

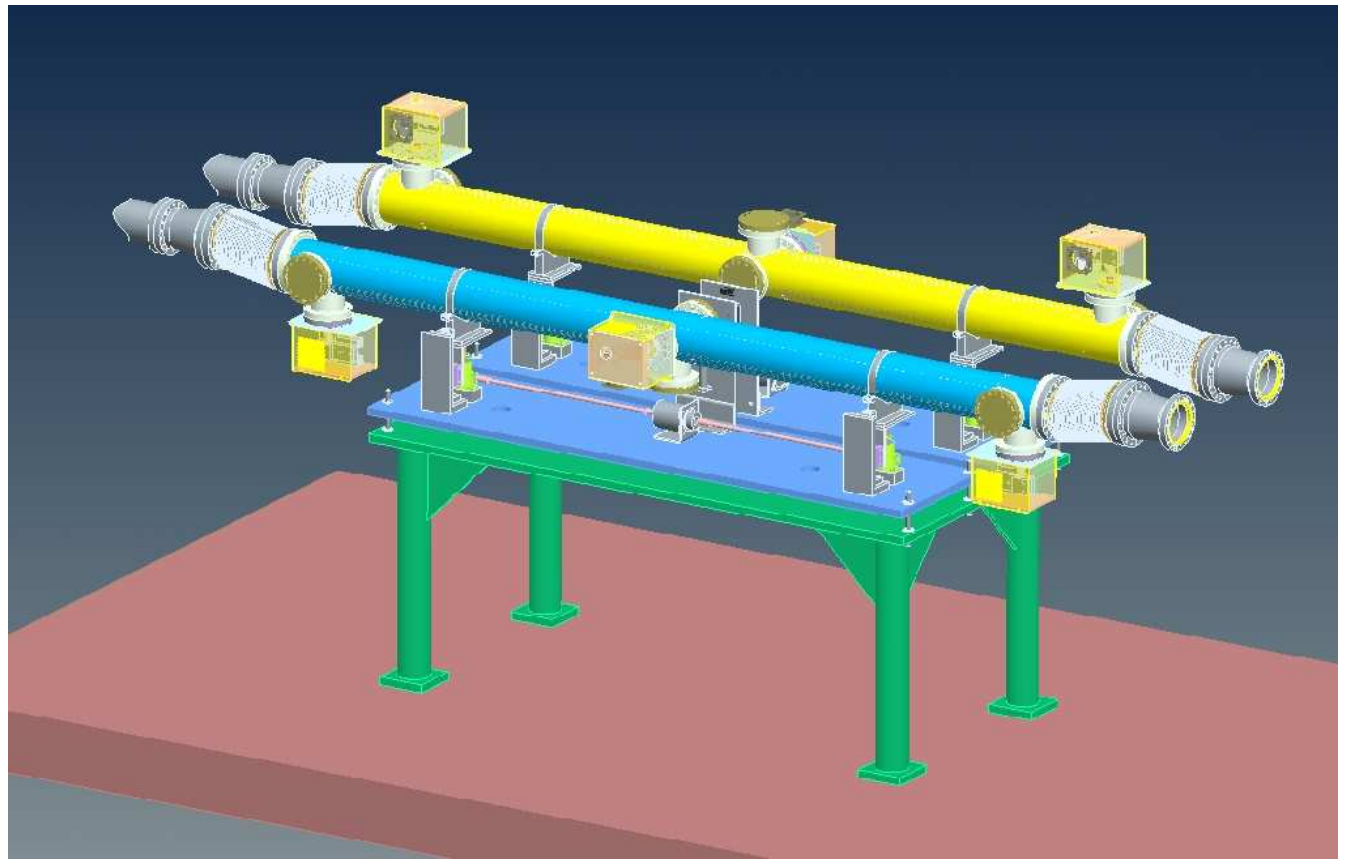

Figure 9: View of two long-range beam-beam compensators in the RHIC ring, mounted on a vertically movable stand. To the right the triplet cryostat is visible, under the beam pipes is a cable tray.

\subsection{Integrated strength}

To compensate a single long-range interaction, the compensator's integrated strength $(I L)$ must be the same as a bunch's current integrated over its length:

$$
(I L)=N_{b} e c,
$$

where $I$ is the current in the wire, $L$ its length, $N_{b}$ the bunch intensity, $e$ the elementary charge, and $c$ the speed of light. For a single long-range interaction with bunches of $N_{b}=2 \times 10^{11}$ protons we have $(I L)=9.6 \mathrm{Am}$. The wire compensator parameters are summarized in Tab. 3.

In the LHC, with $25 \mathrm{~ns}$ bunch spacing and a full crossing angle of $300 \mu \mathrm{m}$, an integrated strength of $80 \mathrm{Am}$ is required to correct for the 16 long-range interactions on either side of an interaction region [4]. Such a strength is also expected to lead to enhanced diffusion at separation amplitudes larger than $6 \mathrm{rms}$ transverse beam sizes. [12]. The RHIC wire compensator will be designed for an integrated strength of 125 Am to allow for the observation of enhanced transverse diffusion in experiments. 
Table 3: Main parameters for RHIC long-range beam-beam compensators.

\begin{tabular}{lccc}
\hline \hline quantity & unit & value & comment \\
\hline integrated strength $(I L)$, single interaction & $\mathrm{Am}$ & 9.6 & \\
maximum integrated strength $(I L)_{\max }$ & $\mathrm{Am}$ & 125 & \\
length of wire $L$ & $\mathrm{~m}$ & 2.5 & \\
radius of wire $r$ & $\mathrm{~mm}$ & 3.5 & \\
number of heat sinks $n$ & $\ldots$ & 3 & at both ends and in middle \\
electrical resistivity $\rho_{e}$ & $\Omega_{\mathrm{m}}$ & $1.72 \times 10^{-8}$ & $\mathrm{Cu}\left(\right.$ at $\left.20^{\circ} \mathrm{C}\right)$ \\
heat conductivity $\lambda$ & $\mathrm{Wm} \mathrm{K}^{-1}$ & 384 & $\mathrm{Cu}\left(\right.$ at $\left.20^{\circ} \mathrm{C}\right)$ \\
density $\rho_{g}$ & $\mathrm{~kg} / \mathrm{m}^{3}$ & $8.96 \times 10^{3}$ & $\mathrm{Cu}\left(\right.$ at $\left.20^{\circ} \mathrm{C}\right)$ \\
thermal expansion coefficient & $\mathrm{K}$ & $1.68 \times 10^{-5}$ & $\mathrm{Cu}\left(0\right.$ to $\left.100^{\circ} \mathrm{C}\right)$ \\
melting temperature & $\mathrm{K}$ & 1083 & $\mathrm{Cu}$ \\
radius of existing beam pipe $r_{p}$ & $\mathrm{~mm}$ & 60 & \\
\hline current in wire $I$, single interaction & $\mathrm{A}$ & 3.8 & \\
maximum current in wire $I_{\max }$ & $\mathrm{A}$ & 50 & \\
electric resistance $R$ & $\mathrm{~m} \Omega$ & 1.12 & \\
maximum voltage $U_{\max }$ & $\mathrm{mV}$ & 55.9 & \\
maximum dissipated power $P_{\max }$ & $\mathrm{W}$ & 2.8 & \\
maximum temperature change $\Delta T_{\max }$ & $\mathrm{K}$ & 15 & \\
maximum change in length $\Delta L_{\max }$ & $\mathrm{mm}$ & 0.4 & \\
vertical position range & $\mathrm{mm}$ & 65 & for $\gamma=107, \beta^{*}=1 \mathrm{~m}$, \\
vertical position range & $\sigma_{y}$ & 10.6 & \\
weight of wire $G$ & $\mathrm{~kg}$ & 0.9 & \\
\hline \hline
\end{tabular}

\subsection{Wire temperature in operation}

A major concern in the design of the long-range compensator is the extraction of the heat dissipated in the wire. The wire's temperature should not exceed $100^{\circ} \mathrm{C}$ to avoid increased outgassing of the vacuum components. Since the wire is located in vacuum it can only be cooled through heat sinks connected to the outside of the chamber, or through an internal cooling channel. Radiation cooling is negligible. Internal water cooling was chosen for the SPS units. We will use heat sinks cooled with forced air.

With $n$ heat sinks, spaced apart by $L /(n-1)$, the maximum temperature increase in the center between 2 heat sinks is [13]

$$
\Delta T_{\max }=\frac{1}{8 \pi^{2}} \frac{\rho_{e}}{\lambda} \frac{(I L)^{2}}{(n-1)^{2} r^{4}}
$$

where $\rho_{e}$ is the electrical resistivity, $\lambda$ the heat conductivity, and $r$ the radius of the wire. Note that $T_{\max }$ only depends on the integrated strength $(I L)$, not the length $L$, and that a large radius and multiple heat sinks are advantageous. In order to move the wire compensator close to the beam, its radius should not be much larger than an rms transverse beam size $\sigma$. Tab. 4 shows lattice functions and beam sizes at both the long-range interaction and the compensator location. We chose a radius of $r=3.5 \mathrm{~mm}$, smaller than a vertical rms beam size for all lattices. This leads to a temperature increase 
Table 4: Lattice functions and beam sized at the locations of the long-range beam-beam interaction and the compensator for different lattices. In all cases a normalized $95 \%$ emittance of $\epsilon_{n}=15 \mathrm{~mm} \cdot \mathrm{mrad}$ is assumed.

\begin{tabular}{lccccc}
\hline \hline & $\begin{array}{c}\text { location } s \\
{[\mathrm{~m}]}\end{array}$ & $\begin{array}{c}\beta_{x} \\
{[\mathrm{~m}]}\end{array}$ & $\begin{array}{c}\beta_{y} \\
{[\mathrm{~m}]}\end{array}$ & $\begin{array}{c}\sigma_{x} \\
{[\mathrm{~mm}]}\end{array}$ & $\begin{array}{c}\sigma_{y} \\
{[\mathrm{~mm}]}\end{array}$ \\
\hline \multirow{3}{*}{$\beta^{*}=10 \mathrm{~m}}$, & $E=24.3 \mathrm{GeV}$ \\
Long-range interaction & 10.6 & 21 & 21 & 1.5 & 1.5 \\
Compensation & 41 & 40 & 127 & 3.3 & 5.9 \\
\hline \multirow{4}{*}{$\beta^{*}=1.0 \mathrm{~m}}$, & $E=100 \mathrm{GeV}$ \\
Long-range interaction & 10.6 & 114 & 114 & 1.6 & 1.6 \\
Compensation & 41 & 395 & 1143 & 3.0 & 5.2 \\
\hline \multirow{3}{*}{$\beta^{*}=0.5 \mathrm{~m}}$, & $E=250 \mathrm{GeV}$ \\
Long-range interaction & 10.6 & 225 & 235 & 1.5 & 1.5 \\
Compensation & 41 & 640 & 1860 & 2.5 & 4.2 \\
\hline \hline
\end{tabular}

of $T_{\max }=15 \mathrm{~K}$ for the maximum considered compensator strength of $(I L)_{\max }=125 \mathrm{Am}$, and 3 heat sinks (see Tab. 3).

Due to the temperature change, the length of the wire will change by

$$
\Delta L=\frac{2}{3} \alpha L \Delta T_{\max }
$$

where $\alpha$ is the thermal expansion coefficient. For our parameters we obtain $\Delta L_{\max }=$ $0.4 \mathrm{~mm}$. The electrical and thermal feedthroughs, insulated with ceramic material, cannot take any mechanical stress and must therefore be connected to the wire through flexible transitions. Fig. 10 shows a design for the electrical feed-through and fan for cooling to ambient temperature. Fig. 9 depicts the end connection with a loop to avoid mechanical stress on the ceramic feedthrough insulator.

\subsection{Positioning range and accuracy}

To avoid any interference with the normal operation of RHIC, the retracted wire compensator should be in the shadow of the existing beam pipe with a radius of $r_{p}=60 \mathrm{~mm}$. The compensator should also be able to approach the beam to $3 \sigma$ or less. A range of the vertical position of $65 \mathrm{~mm}$ is sufficient to accomplish this, with the closest position surveyed to coincide with the nominal beam trajectory. Large bellows are needed to allow for the vertical positioning range.

We plan to allow for vertical motion only, having the angle surveyed to be the same as the nominal beam trajectory. A positioning accuracy of about $0.1 \sigma$ or $0.15 \mathrm{~mm}$ is expected to sufficient. In Ref. [12] transverse distance errors between 0 and $20 \%$ were found to be acceptable in simulations. Fig. 9 also shows the strong back to reduce the wire sagging to less than $0.1 \sigma$. The wire is supported at 7 locations.

With the gear under load the accuracy should be better than the manufacturer specified slack of $0.25 \mathrm{~mm}$. We note that the weight of the wire is

$$
G=\pi r^{2} L \rho_{g},
$$




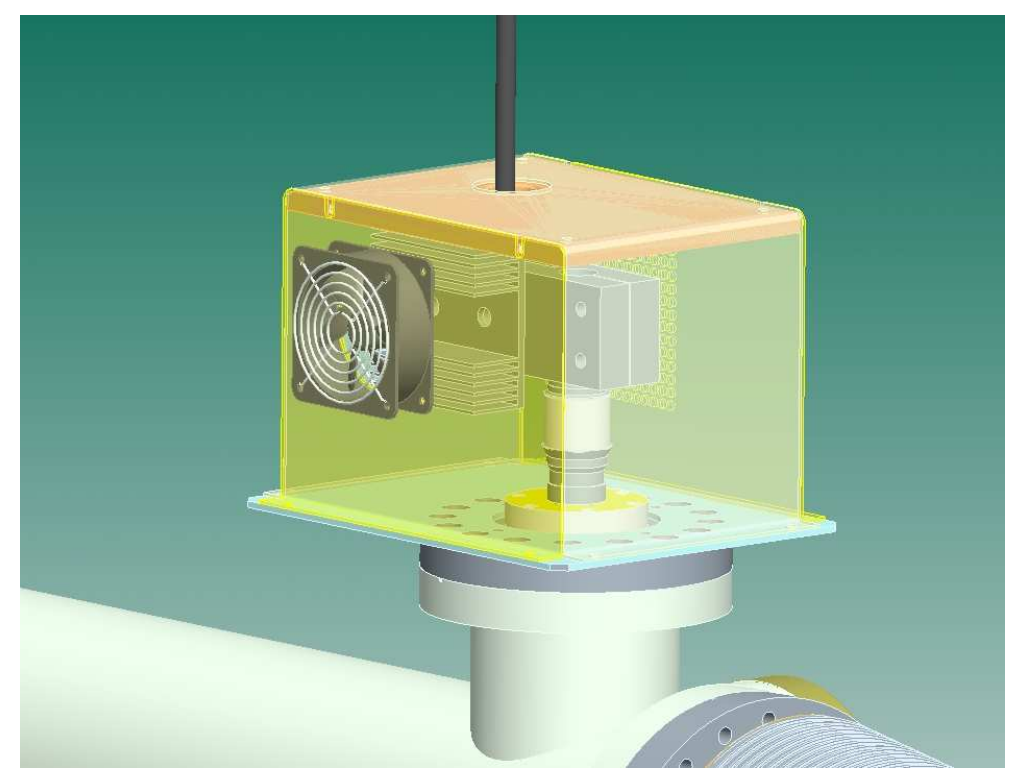

Figure 10: Connection box with electrical feed-through and fan for cooling to ambient temperature.

where $\rho_{g}$ is the density. With the parameters in Tab. 3 we have $G=0.9 \mathrm{~kg}$. This is only a fraction of the total assembly seen in Figs. 9 and 10.

\subsection{Power supply requirements}

The current, resistance, and voltage of the wire are

$$
I=\frac{(I L)}{L}, \quad R=\frac{\rho_{e} L}{\pi r^{2}} \quad \text { and } \quad U=I R
$$

Tab. 3 shows a maximum current of $50 \mathrm{~A}$, and a maximum voltage of $56 \mathrm{mV}$. For the compensation of a single long-range interaction, a current of $3.8 \mathrm{~A}$ is sufficient.

A current ripple of $\Delta I / I<10^{-4}$ is required [12] which can be accomplished with a single power supply for the considered current range. In a second stage a pulsed or modulated power supply can be tested, which is needed for the LHC. Since a $100 \%$ modulation depth is required for the LHC, such a power supply is very challenging to avoid emittance growth from noise.

We make an estimate of the emittance growth due to random dipole kicks generated by current ripples in the compensator. The emittance growth rate due to random dipole kicks is $[14]$

$$
\frac{1}{\tau}=\frac{1}{\epsilon_{n}} \frac{d \epsilon_{n}}{d t}=3 \frac{(\beta \gamma)}{\epsilon_{n}} f_{r e v} \beta_{0} \theta_{r m s}^{2}
$$

where $\epsilon_{n}$ is the nomalized $95 \%$ emittance, $\beta_{0}$ is the $\beta$-function at the compensator location, and $\theta_{r m s}$ is the rms steering error. The magnetic field at distance $d$ from the wire, and 


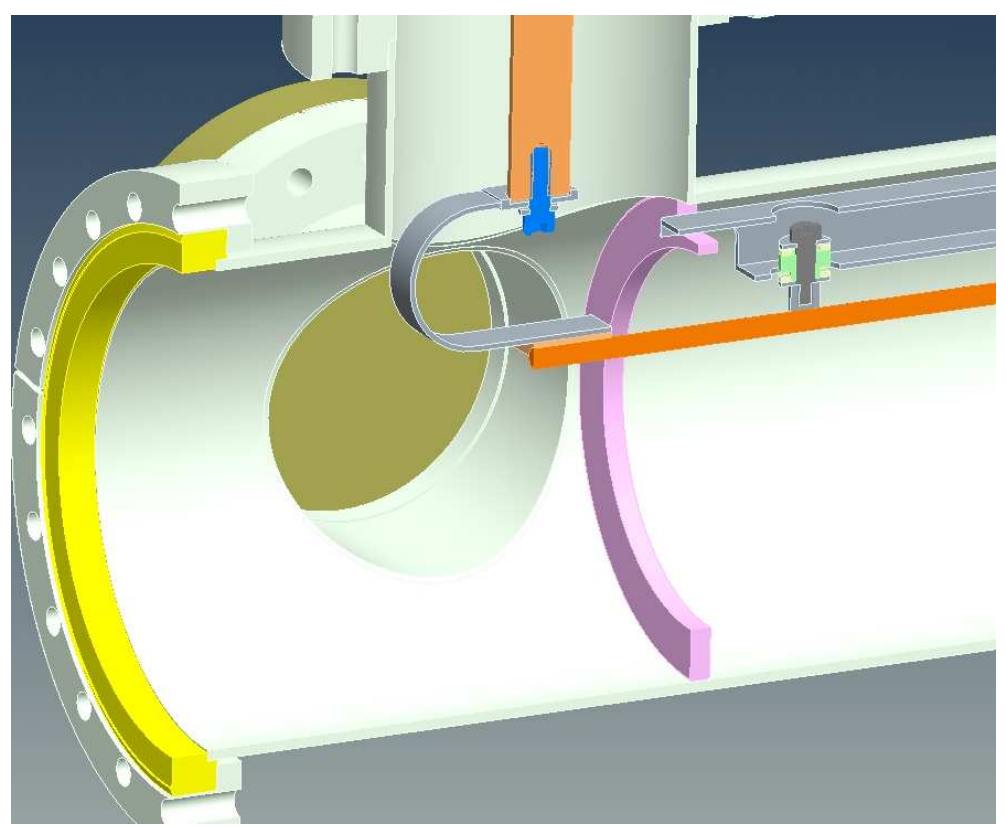

Figure 11: Wire end connection with loop to avoid mechanical stress on the ceramic feedthrough insulator. Also seed is the strong-back to reduce the wire sagging.

its integrated strength is

$$
B=\frac{\mu_{0} I}{2 \pi d} \quad \text { and } \quad(B L)=\frac{\mu_{0} I L}{2 \pi d}
$$

where $\mu_{0}$ is the permeability of the vacuum. This makes a dipole kick of angle

$$
\theta=\frac{(B L) e}{2 \pi p}=\frac{\mu_{0}}{(2 \pi)^{2}} \frac{e}{p} \frac{L}{d} I
$$

where $e$ is the elementary charge, and $p$ the proton momentum. For a beam energy of $100 \mathrm{GeV}$, and and emittance of $20 \mathrm{~mm} \cdot \mathrm{mrad}$, we have

$$
\frac{1}{\tau} \approx 3.5 \times 10^{-4} \frac{m^{2}}{A^{2} s} \frac{I_{r m s}^{2}}{d^{2}} .
$$

At a distance of $d=3 \sigma$, and requiring an emittance growth time of $100 \mathrm{~h}$, it follows $I_{r m s}<1.6 \mathrm{~mA}$, or $I_{r m s} / I<4 \times 10^{-4}$ for the single bunch correction. We note, however, that modulational effects can be more harmful than such a simple calculation indicates, since the magnetic field of the wire is nonlinear.

\subsection{Controls and diagnostics}

Control is needed for the power supply (on and off) and the current, as well as the collimator motion. The motion control will be similar to a collimator. It is planned to install additional PIN diodes as loss monitors in the vicinity of the long-range compensators. 


\section{Future experiments}

So far the long-range effect was only tested at injection. Further tests of the long-range effect could include the observation of beam loss rates and emittance growth as a function of vertical separation

- At $100 \mathrm{GeV}$ and possibly $250 \mathrm{GeV}$

- At various transverse tunes

- With more than one long-range interactions

- With multiple long-range interactions of varying phase advance

Once the long-range compensators are operational their effects can be tested

- With varying distance between the beam and the compensator

- With varying phase advance between the long-range interaction and the compensator

- With multiple long-range interactions of varying phase advance and a single compensator

In addition, the onset of enhanced diffusion may be observed with a compensator strength about an order of magnitude stronger than needed for a single bunch compensation. The compensator is designed for such a strength (see Sec. 3).

A better understanding of the beam lifetime and emittance growth of stored proton beams in RHIC would allow a more accurate assessment of the effect of long-range beambeam interactions. This is investigated in a separate report.

\section{Summary}

A long-range beam-beam effect is observable with single bunches in RHIC at injection. The beam loss rate is sensitive to both the transverse separation and the tunes. A long-range wire compensator is being designed for RHIC, and it is planned to install 2 compensators in 2006. Before the wire compensators can be installed, it is planned to measure the beam loss rate as a function of the vertical separation also at the current RHIC store energy of $100 \mathrm{GeV}$. Beam tests with the wire compensator could begin in Run-7.

\section{Acknowledgments}

The authors are thankful to R. Calaga, A. Drees, T. Roser, S. Tepikian, J. Tuozzolo, and V. Ptityn for comments. 


\section{References}

[1] V. Shiltsev, V. Danilov, D. Finley, and A. Sery, "Considerations on compensation of beam-beam effects in the Tevatron with electron beams", Phys. Rev. ST Accel. Beams 2, 071001 (1999).

[2] V. Shiltsev and A. Zinchenko, "Electron beam distortions in beam-beam compensation setup", Phys. Rev. ST Accel. Beams 1, 064001 (1998).

[3] J.P. Koutchouk, "Principle of a correction of the long-range beam-beam effect in the LHC using electromagnetic lenses", CERN LHC Project Note 233 (2000).

[4] J.-P. Koutchouk, "Correction of the long-range beam-beam effect in LHC using electromagnetic lenses", proceedings of the 2001 Particle Accelerator Conference, Chicago; SL Report 2001-048 (2001).

[5] J.P. Koutchouk, "Experiments on LHC long-range beam-beam compensation in the SPS", proceedings of the 2004 European Particle Accelerator Conference, Lucerne (2004).

[6] B. Erdelyi and T. Sen, "Compensation of beam-beam effects in the Tevatron with wires", FNAL-TM-2268 (2004).

[7] B. Erdelyi and T. Sen, "Feasibility study of beam-beam compensation in the Tevatron with wires", proceedings of the 2005 Particle Accelerator Conference, Knoxville, Tennessee (2005).

[8] F. Wang, M. Farkhondeh, W. Franklin, W. Graves, R. Milner, J. Van der Laan, C. Tschalaer, D. Wang, T. Zwart, A. Zolfaghari, J. Beebe-Wang, A. Desphande, V. Litvinenko, W.W. MacKay, C. Montag, B. Parker, S. Peggs, S. Ozaki, V. Ptitsyn, T. Roser, S. Tepikian, D. Trbojevic, "The eRHIC ring-ring collider design", proceedings of the 2005 Particle Accelerator Conference, Knoxville, TN (2005).

[9] S. Peggs, "Parasitic beam-beam collisions and crossing angles in RHIC", proceedings of the Beam-Beam'99 workshop, CERN; BNL RHIC/AP/169 (1999).

[10] F. Zimmermann, "Beam-beam compensation schemes", proceedings of the First CARE-HHH-APD Workshop on Beam Dynamics in Future Hadron Colliders and Rapidly Cycling High-Intensity Synchrotrons (HHH-2004), CERN, Geneva, Switzerland, CERN-2005-006, p. 101 (2005).

[11] F. Zimmermann, P. Lebrun, T. Sen, V. Shiltsev, X.L. Zhang, "Using the Tevatron electron lens as a wire and other TEL studies at FNAL", CERN AB-Note-2004-041 (2004).

[12] F. Zimmermann, "Weak-strong simulation studies for the LHC long-range beambeam compensation", proceedings of the Beam-Beam Workshop at Fermilab, FERMILAB-Conf-01/390-T, CERN LHC Project Report 502 (2001) 
[13] J.-P. Koutchouk, G. de Rijk, F. Zimmermann, "Simulation of the LHC Long-Range Beam-Beam Effect at the SPS", Draft Note, (2002).

[14] M. Syphers, "Emittance dilution effects [1]", in "Handbook of accelerator physics and engineering", World Scientific, (1998). 